\title{
Search and rescue (SAR) operations for the missing Bell 206 Long Ranger helicopter in Sarawak, Malaysia using near real-time airborne hyperspectral imaging system
}

\begin{abstract}
Purpose - The aim of this paper is to present the latest advances in real-time airborne hyperspectral sensing applications in identifying and mapping the likely spots to be zeroed in for the SAR operations. Design/methodology/approach - A Sabah Air GAF Nomad N22B low altitude fixed wing aircraft equipped with an AISA airborne hyperspectral imaging system flew over the steep gradient carved by very narrow valleys, ridges, precipitous escarpments and ravines, extensively covered by thick virgin forest of the highlands. The study was carried out by an AISA sensor, which is a complete system that consists of a compact hyperspectral sensor head, miniature GPS/INS sensor for precise positioning, data acquisition unit and Caligeo post-processing software. Findings - These UPM-APSB's AISA flights demonstrated that quality real-time hyperspectral images could be pre-processed onboard the aircraft and become accessible to the SAR committee members for quick ground SAR within two hours after the flight. The combined geospatial information technologies were a major breakthrough in the Malaysian SAR real-time imaging technique. Originality/value - It is expected that future precise locations of the suspected targets can be transmitted via very high frequency radio communications and become accessible to the SAR ground members such as the commandos VAT 69. Real-time airborne hyperspectral imaging will benefit strategic SAR and help reduce the loss of lives in future helicopter crashes in the Bario-Ba'Kelalan areas.
\end{abstract}

Keyword: Forests, Helicopters, Malaysia, Search and rescue, Sensors 